\title{
Analyzing asymmetric effects of cryptocurrency demand on environmental sustainability
}

\author{
Sinan Erdogan ${ }^{1}$ - $\cdot$ Maruf Yakubu Ahmed ${ }^{2}$. Samuel Asumadu Sarkodie ${ }^{2}$
}

Received: 29 September 2021 / Accepted: 3 December 2021 / Published online: 11 January 2022

(C) The Author(s), under exclusive licence to Springer-Verlag GmbH Germany, part of Springer Nature 2021

\begin{abstract}
When bitcoin (BTC), the first pioneering cryptocurrency was released in 2009, it was considered an apolitical currency. Besides, the possible effect of BTC and other cryptocurrencies on either financial markets or transactions has been widely discussed. However, the environmental effects of cryptocurrency demand have been ignored. Here, this study examines the nexus between cryptocurrencies and environmental degradation by employing standard and asymmetric causality methods. The Toda-Yamamoto and bootstrap-augmented Toda-Yamamoto test results reveal Bitcoin and Ethereum (ETH) excluding Ripple (XRP) have causal effects on environmental degradation. The Fourier-augmented Toda-Yamamoto test results show causal effects running from Bitcoin and Ripple to environmental degradation, whereas no causal effect runs from Ethereum to environmental degradation. The asymmetric causality shows causal effects from the positive shock of Bitcoin demand, negative shocks of Ripple and Ethereum demands to positive shocks of environmental degradation. Further discussions and policy implications are provided in the relevant sections of this study.
\end{abstract}

Keywords Environmental sustainability · Cryptocurrency · Asymmetric causality · Bitcoin (BTC) · Ethereum (ETH) · Ripple (XRP)

\section{Introduction}

The demand for energy to mine and transact cryptocurrencies has triggered global debate in recent years. Cryptocurrency emissions, especially bitcoin is reported to push global warming above $2{ }^{\circ} \mathrm{C}$, hence, causing uproar among academicians, mainstream media, and the public (Mora et al. 2018). While studies in this scope are topical, the existing literature appears to focus

Responsible Editor: Philippe Garrigues

Sinan Erdogan

phderdogan@gmail.com

Maruf Yakubu Ahmed

ahmedmyakubu@gmail.com

Samuel Asumadu Sarkodie

asumadusarkodiesamuel@yahoo.com

1 Department of Economics, Faculty of Economics and Administrative Sciences, Hatay Mustafa Kemal University, 31060 Hatay, Turkey

2 Nord University Business School (HHN), Post Box 1490, 8049 Bodø, Norway on bitcoin, ignoring alternative coins. Thus, assessing the effect of several cryptocurrencies on emissions expands the global debate with policy implications. The technological innovation behind peer-to-peer electronic currency, viz. blockchain is considered one of the greatest innovations in the twenty-first century. The first blockchain technology namely bitcoin was proposed by Satoshi Nakamoto in his 2008 white paper. Bitcoin is a decentralized digital electronic currency that enables online payment without recourse to any intermediary. Since its inception, thousands of second generational cryptocurrencies aka alternative coins have emerged including Ethereum, Binance coin, Solana, Cardano, Ripple, and Polkadot. Currently, the estimated total cryptocurrency market capitalization stands at \$2.51 trillion [est. November 27, 2021] (CoinMarketCap 2021). There are more than 2500 cryptocurrencies in global circulation that are generally based on blockchain technology (Goodkind et al. 2020). Mining is the process by which new mineable tokens of cryptocurrency enter circulation, whereas miners are participants that perform cryptographic work that adds new transactions to the ledger (Kosba et al. 2016). For instance, Nakamoto's paper explains in detail the working principles of bitcoin mining 
which began in 2009 (Nakamoto 2008). Mining requires substantial electricity to power special computers that uses proof-of-work consensus algorithm to validate transactions that are stored in the publication transaction ledger. It is unclear the amount of energy cryptocurrencies consume, as by design, they are hard to track. But the consensus mechanism used by bitcoin for mining and transacting is energy-intensive. For instance, a report by the University of Cambridge Centre for Alternative Finance (CCAF) shows the estimated annual bitcoin total energy consumption between 40 and $445 \mathrm{TWh}$ is similar to the annual energy used by the Netherlands (Rowlatt 2020). Increase in the price of cryptocurrency such as bitcoin spur energy utilization due to computing power used in creating and mining transactions-this has been the weakness of the cryptocurrency industry (Rowlatt 2020). Additionally, the halving of bitcoin rewards and hashing power added to the global mining network drastically increases the mining difficulty and production cost-which may positively influence the price.

Recently, the CEO of Tesla Motors, Elon Reeve Musk announced the suspension of using bitcoin as mode of purchasing Tesla vehicles-citing unsustainable use of fossil fuel energy (i.e., coal) for mining and transacting bitcoin.

The tweet reads,

"Tesla has suspended vehicle purchases using Bitcoin.

We are concerned about rapidly increasing use of fos-

sil fuels for Bitcoin mining and transactions, especially coal, which has the worst emissions of any fuel. Cryptocurrency is a good idea on many levels, and we believe it has promising future, but this cannot come at great cost to the environment. Tesla will not be selling any Bitcoin and we intend to use it for transactions as soon as mining transitions to more sustainable energy. We are also looking at other cryptocurrencies that use $<1 \%$ of Bitcoin's energy/transaction. (Elon 2021)"

Although energy consumption attributed to cryptocurrency market has gained much interest in recent years, however, literature is limited in the scope of assessing the effects of mining and transacting cryptocurrencies on environmental degradation. Motivated by this, we extend the literature to investigate the impact of cryptocurrencies on environmental degradation using the novel standard and asymmetric causality tests. Insights from this study may be helpful to the rapidly growing blockchain technology and management of carbon emissions in the bid to mitigate climate change. Holistic understanding of major cryptocurrencies investigated in this study will inform policy makers in setting up regulations for blockchain and cryptocurrency applications. Unlike other studies that focused only on bitcoin, this study includes Ethereum and Ripple which may provide additional evidence on the environmental damage by alternative coins. The study may inform new crypto developers to create energy-efficient algorithms and green alternatives with less energy requirement throughout the lifecycle.

\section{Literature review}

The criticism of bitcoin is generally centered around the proof-of-work carbon emissions without considering its market value. The estimation of carbon footprint of cryptocurrencies have received considerable attention in both academic and public sphere (Baur and Oll 2021). As with any new emerging technology, careful consideration of its environmental and health impacts ought to be investigated. In recent years, emerging literature investigating these impacts—-such as energy consumption and carbon emissions attributable to cryptocurrency mining including Ethereum, Litecoin, Bitcoin, and Monero-were responsible for 3-15 million tons of carbon emissions (Krause and Tolaymat 2018).

An attempt to use IP addresses to assess mining locations indicate significant carbon emissions associated with bitcoin mining, contributing more than 20 million tons annually (Stoll et al. 2019). It is reported that carbon emissions associated with bitcoin mining alone could exacerbate global anthropogenic emissions. The empirical evidence suggests $\sim 13$ million metric tons of carbon emissions can be attributed to bitcoin blockchain technology expansion between the period spanning 2016-2018 (Krause and Tolaymat 2018). Evidence from the study indicates cryptocurrency mining could be associated with 3-15 million tons of global carbon emissions (Kahn 2018). But the transaction per carbon emitted differs by country-for instance, numerous mining centers based in China are due to low setup cost, and heavy reliance on coal energy - which is, unfortunately, the most carbon-intensive place for mining cryptocurrencies (Jiang et al. 2021). The volatility movement in cryptocurrency prices and the need to minimize operational costs including the cost of energy in order to maximize profit are important drivers in selecting a crypto mining site (Peck 2017). The use of powerful computers requires large energy sources with machines running continuously. A typical computer server that consumes about 1.5 kilowatts $(\mathrm{kW})$ of power may cost between $\$ 3224$ and $\$ 9000$ for mining an individual coin (McGeeham 2018). Existing findings show that without a direct policy intervention in bitcoin blockchain mining, energy consumption in China is estimated to reach $296.59 \mathrm{TWh}$ with a corresponding 130.50 million tons of carbon emissions in 2024 (Jiang et al. 2021). However, the amount of renewable energy sources in Canada is higher relative to China, and similar amount of cryptocurrency mined in China may generate four times carbon emissions compared to Canada (Krause and Tolaymat 2018). It is noteworthy that miners are mainly concerned with the amount of profit obtained than energy efficiency. Hence, 
as more people driven by profit enter the blockchain industry, energy consumption is estimated to increase considerably (Badea and Mungiu-Pupăzan 2021).

Energy utilization in the mining of cryptocurrency is significant and growing at alarming rate. Evidence from a recent study reported that the bitcoin power consumption in the year 2020 amounted to 120 gigawatts per second which is approximately $63 \mathrm{TWh} /$ year (Mohsin 2021). A study that employed the vector error correction model (VECM) reported the short- and long- run bidirectional causality between cryptocurrency volume and environmental degradation (Mohsin et al. 2020). Empirical findings using the novel ARDL reveal the volume of trading all cryptocurrencies has significant positive effect on energy consumption in both short and long run-with long-term consequence on the environment and energy sector (Schinckus et al. 2020). It is reported that the reduction in production cost drivers of cryptocurrencies that improves energy efficiency of mining hardware, have cheaper worldwide electricity prices, and lower mining difficulty could indirectly have a negative impact on the price of cryptocurrencies (Hayes 2017). A study suggests the average mining efficiency was $500 \mathrm{~W}$ per GH/s throughout 2010-2013 (Garcia et al. 2014). However, another study recorded the average mining energy efficiency across different mining networks around $0.40 \mathrm{~W}$ per $\mathrm{GH} / \mathrm{s}$ (Hayes 2017). The study argued that cryptocurrency mining consumes significant amount of energy for proof-of-work to add new blocks to the chain. Each $\$ 1$ Bitcoin value created is reported to cause health and climate damages of $\$ 0.49$ and $\$ 0.37$ in the USA and China, respectively (Goodkind et al. 2020). Other empirical evidence found that the trading volume of all cryptocurrency have significant positive impact in both short and long run on energy consumption (Schinckus et al. 2020). Comparatively, evidence from the existing literature shows that conventional mining of gold, aluminum, copper, and rear earth oxides consumed less energy per 1 US dollar of energy in relation to cryptocurrency mining to produce equivalent market value (Krause and Tolaymat 2018).

The empirical literature assessed thus far appears to focus more on Bitcoin, ignoring the contribution of alternative coins to both energy and environmental accounting. While Bitcoin accounts for $42 \%$ of the entire crypto market valuation, alternative coins namely second and third generation account for $58 \%$; hence, the inclusion of alternative coins alongside Bitcoin in a carbon function contributes to the global debate on environmental sustainability.

\section{Data, methodology, and empirical results}

The environmental effect of economic development has been increasing since the Industrial Revolution. Awareness on the exacerbating effects of economic activities on environmental quality has been an increasing in the last decades. In this regard, the number of empirical research in economics and environment is increasing enormously. The seminal work of Grossman and Krueger (1991) has improved the empirical studies on environment and economics nexus. Earlier studies have focused on the effect of economic development on environmental degradation, and international trade; yet, the other studies have focused on the effect of other economic determinants of environmental pollution including energy-based variables, institutional variables, domestic investments, foreign direct investments, urbanization, population, and financial development (Akalin et al. 2021; Danish and Ulucak 2020; Erdogan 2020; Ilkay et al. 2021 Sinha et al. 2019). Based on these theoretical discussions, we included cryptocurrency demand to investigate cryptocurrency demand-environment nexus on a global level. The general form of employed logarithmic models for the period 2010(M8) to 2021 (M1) to unveil the causal effects between cryptocurrency demand and environmental pollution can be expressed as follows ${ }^{1}$ :

$\mathrm{CO} 2_{t}=\alpha+\beta_{1} C R_{t-1}+\ldots+\beta_{p+d} C R_{t-(p+d)}+\varepsilon_{t}$

$\mathrm{CO} 2_{t}^{ \pm}=\theta+\delta_{1} C R_{t-1}^{ \pm}+\ldots+\delta_{p} C R_{t-1}^{ \pm}+v_{t}^{ \pm}$

where $\mathrm{CO}_{2}$ denotes global carbon emission whereas $C R$ represents cryptocurrency demand, respectively. Due to lack of data measurement, we used monthly data of global carbon dioxide emissions (parts per million) from 2010 (M8) to 2021 (M1), and monthly prices of -Bitcoin (BTC) from 2010 (M8) to 2021 (M1), Ripple (XRP) 2015 (M2) to 2021 (M1), and Ethereum (ETH) from 2016 (M4) to 2021 (M1) to test causality among cryptocurrency demand and environmental degradation. According to Investing (2021), the daily trade volume of the total cryptocurrency trade is approximately 1.562 billion U.S. Dollars (USD), whereas the daily trade volume of BTC, XRP, and ETH is almost 1.166 billion USD. In this manner, the share of BTC, XRP, and ETH is approximately $71.96 \%$ of total traded cryptocurrencies. Besides, BTC, XRP, and ETH are among the oldest and well-known cryptocurrencies; thus, cryptocurrency demand was proxied by using the prices of BTC, XRP, and $\mathrm{ETH}$, while data on carbon dioxide emissions were used as indicator of environmental degradation. Data on carbon dioxide emissions were obtained from Global Monitoring Laboratory (2021), whereas cryptocurrency price data were obtained from Investing (2021). In Fig. 1, we applied seasonal-adjustment procedures to the monthly data using

\footnotetext{
${ }^{1}$ Equation 1 represents general form of Toda-Yamamoto and Fourier Toda-Yamamoto causality approaches, while Eq. 2 represents general form of asymmetric causality approach.
} 

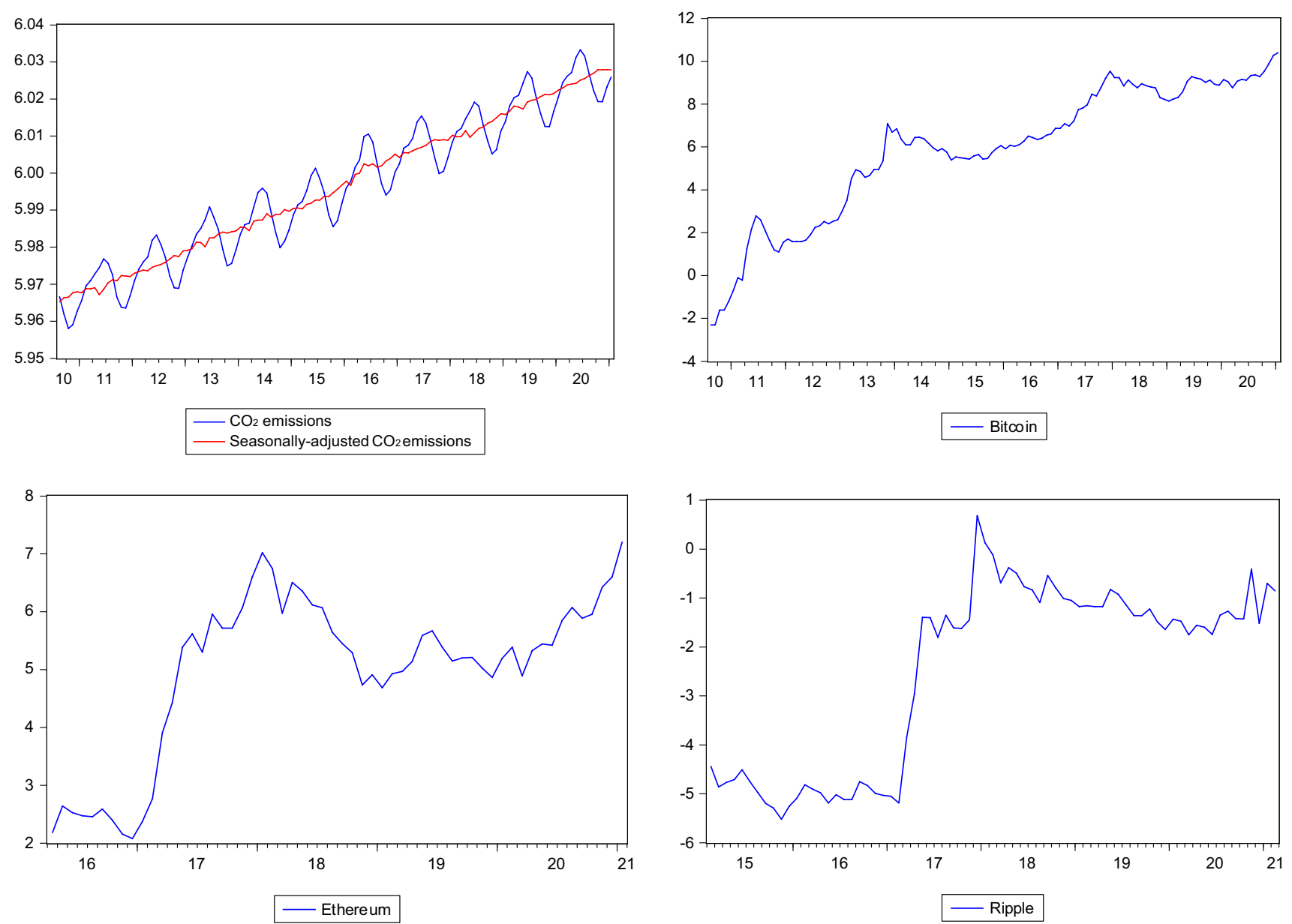

Fig. $1 \mathrm{CO}_{2}$ Emission from 2010 (M8) to 2021 (M1) and cryptocurrency prices

Census X-13 procedure. The trend of carbon dioxide emissions shows seasonality as expected. All variables were converted into logarithmic values.

Second, we used Games-Howell parametric technique (Patil 2021) for hypothesis testing to examine price distribution across sampled cryptocurrencies as depicted in Fig. 2. Advantageously, Games-Howell technique do not consider equal variance of variables in the pairwise comparison test. We further employed omega-squared $\left(\omega^{2}\right)$ function to compute effect size in the estimation procedure. Welch's $F$-test (Welch 1951) in Fig. 2 shows that across 256 cryptocurrency prices, Bitcoin exhibits significantly higher price than both Ethereum ( $p$-value $<0.05)$ and Ripple $(p$-value $<0.01)$. Similarly, Ethereum exhibits significantly higher price ( $p$-value $<0.01)$ than Ripple. Thus, we find significant differences (i.e., $\omega^{2}=0.8395 \%$ CI $[0.80,1.00]$ ) in prices across three cryptocurrencies. This implies BTC and alternative coins (ETH and XRP) will respond differently in price when there is a global shock. The validity of heterogeneous distribution in crypto prices underscores the importance of using novel causality tests to investigate the direction of causality between crypto price volatility and environmental degradation.

We began our analysis by employing the causality test proposed by Toda and Yamamoto (1995). Toda-Yamamoto causality test can be implemented regardless of integration and cointegration properties of the data. Hence, preliminary analysis for determining the integration level of variables and existence of cointegration are not require. TodaYamamoto causality approach is simply based on the idea of estimation following Vector Autoregressive (VAR) model expressed as follows:

$y_{t}=\alpha+\beta_{1} y_{t-1}+\ldots+\beta_{p+d} y_{t-(p+d)}+\varepsilon_{1 t}$

where $y_{t}$ is formed by $K$ endogenous variables, $p$ is the lag length, $d$ is the maximum integration level of the variables, $\alpha$ is the intercept term, $\beta$ are coefficient matrices, and $\varepsilon_{1 t}$ is the error term. The Toda-Yamamoto causality test adopts the null hypothesis of Granger non-causality $\left(H_{0}: \beta_{1}=\ldots .=\beta_{p}=0\right)$, with estimated test statistic based on Wald statistic and asymptotic $\chi^{2}$ distribution with $p$ degrees of freedom (Nazlioglu 
Fig. 2 Log distribution of bitcoin, ethereum and ripple

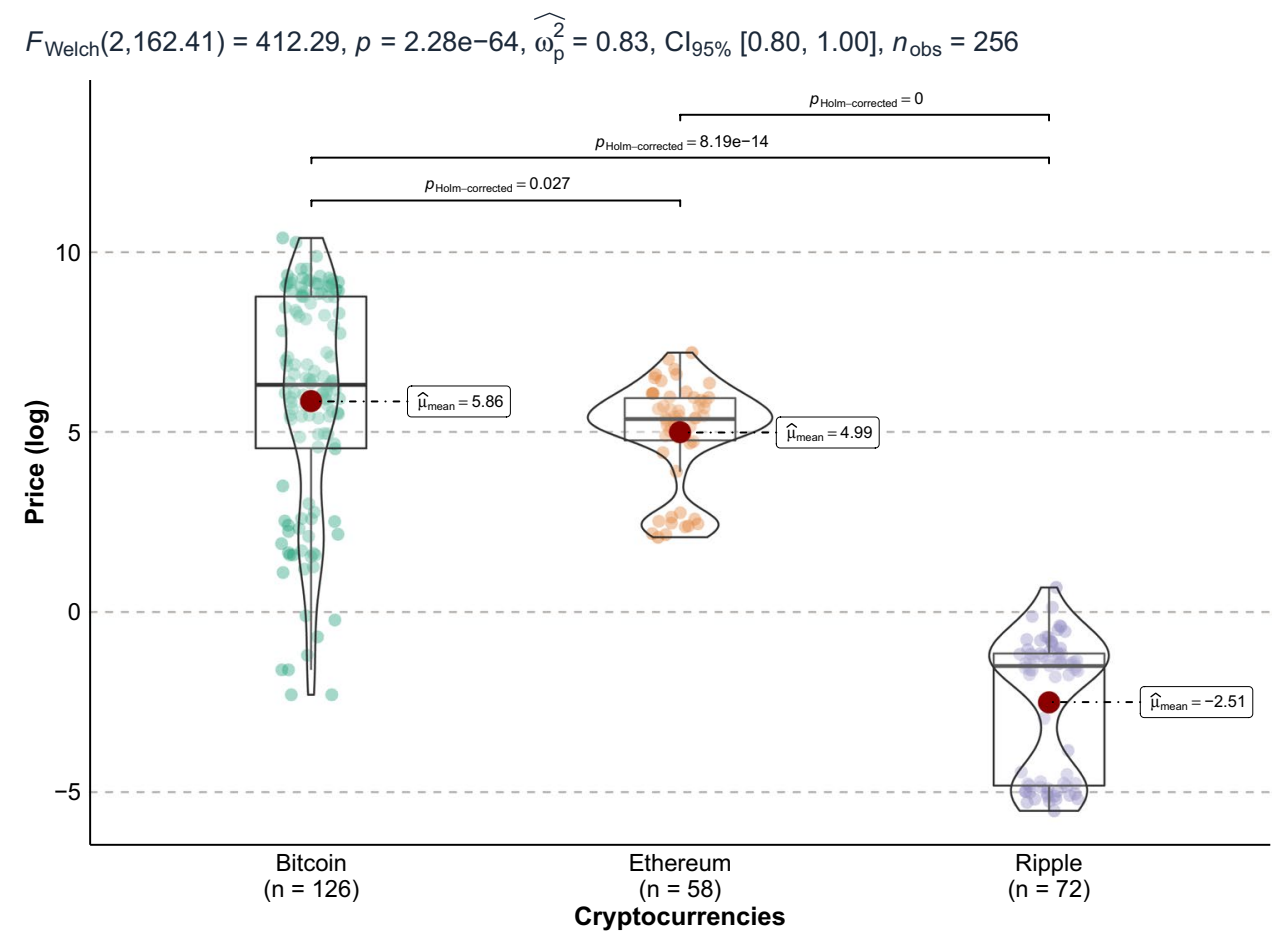

Pairwise test: Games-Howell test; Comparisons shown: only significant et al. 2016; Toda and Yamamoto 1995). However, Hacker and Hatemi-J (2006) reported that size properties of the TodaYamamoto causality approach perform relatively weak in the case of asymptotic distribution $\left(\chi^{2}\right)$. Moreover, utilization of the leveraged bootstrap distribution could give satisfying results on minimizing size distortions. Therefore, proposing the use of bootstrap-augmented Toda-Yamamoto (Hacker and Hatemi-J 2006) causality test.

The economic data generally exhibit structural changes, thus, ignoring such changes could lead to biased inferences in testing hypothesis (Erdogan et al. 2020). Additionally, failure to account for structural changes in data can cause false rejection of the null of non-causality (Enders and Jones 2016). Hence, Nazlioglu et al. (2016) extended the traditional Toda-Yamamoto procedure to include Fourier approximation that control for structural changes. Like the standard Toda-Yamamoto procedure, the Fourier Toda-Yamamoto procedure does not require any prior knowledge on variables including integrational level and cointegration. The data generation procedure of the Fourier Toda-Yamamoto test can be given as (Nazlioglu et al. 2016):

$y_{t}=\alpha(t)+\beta_{1} y_{t-1}+\ldots+B_{p+d} y_{t-(p+d)}+\varepsilon_{2 t}$

where $\alpha(t)$ is a function of time, and represents the structural shifts in the dependent variable. Equation 4 can be extended by using Fourier functions as follows: $\alpha_{t}=\alpha_{0}+\sum_{k=1}^{n} \gamma_{1 k} \sin (2 \pi k t / T)+\sum_{k=1}^{n} \gamma_{2 k} \cos (2 \pi k t / T)$

where $n$ is the number of frequencies. Equation 5 can be modified by including frequency as follows:

$\alpha_{t}=\alpha_{0}+\gamma_{1} \sin (2 \pi k t / T)+\gamma_{2} \cos (2 \pi k t / T)$

where $k$ is the number of Fourier frequency. The general procedure of the Fourier Toda-Yamamoto can be expressed by utilizing Eqs. 4 and 6 as follows:

$y_{t}=\alpha_{0}+\gamma_{1} \sin (2 \pi k t / T)+\gamma_{2} \cos (2 \pi k t / T)+\beta_{1} y_{t-1}+\ldots+B_{p+d} y_{t-(p+d)}+\varepsilon_{2 t}$

Nazlioglu et al. (2016) adopted the null hypothesis of non-Granger causality, and employed bootstrap procedure to obtain more powerful test statistics that satisfies small sample properties. Such technique is robust in the case of the existence of unit root and cointegration. In traditional causality literature, no separation is made between the causal impact of positive and negative shocks, and such an assumption might be hard to satisfy. Hatemi-j (2012) proposed an asymmetric causality approach to address this issue. The data generation process of asymmetric causality test is based on the idea of causality between two integrated variables as follows (Hatemi-j 2012):

$y_{3 t}=y_{3 t}+\varepsilon_{3 t}=y_{30}+\sum_{i=1}^{t} \varepsilon_{3 i}$ 
$y_{4 t}=y_{4 t}+\varepsilon_{4 t}=y_{40}+\sum_{i=1}^{t} \varepsilon_{4 i}$

where $y_{1 t}$ and $y_{2 t}$ are constant, $\varepsilon_{3 i}$ and $\varepsilon_{4 i}$ are residuals. Positive and negative shocks can be defined as $\varepsilon_{3 i}^{+}=\max \left(\varepsilon_{3 i}, 0\right), \varepsilon_{4 i}^{+}=\max \left(\varepsilon_{4 i}, 0\right), \varepsilon_{3 i}^{-}=\max \left(\varepsilon_{3 i}, 0\right), \varepsilon_{4 i}^{-}=\max \left(\varepsilon_{4 i}, 0\right)$, respectively. Hence, $\varepsilon_{3 i}$ and $\varepsilon_{4 i}$ can be extended as $\varepsilon_{3 i}=\varepsilon_{3 i}^{+}+\varepsilon_{3 i}^{-}$and $\varepsilon_{4 i}=\varepsilon_{4 i}^{+}+\varepsilon_{4 i}^{-}$, respectively. Therefore, Eq. 8 and Eq. 9 can be shaped as follows:

$$
\begin{aligned}
& y_{3 t}=y_{3 t-1}+\varepsilon_{3 t}=y_{3,0}+\sum_{i=1}^{t} \varepsilon_{3 i}^{+}+\sum_{i=1}^{t} \varepsilon_{3 i}^{-} \\
& y_{3 t}=y_{4 t-1}+\varepsilon_{4 t}=y_{4,0}+\sum_{i=1}^{t} \varepsilon_{4 i}^{+}+\sum_{i=1}^{t} \varepsilon_{4 i}^{-}
\end{aligned}
$$

The cumulative form of the positive and negative shocks of the variables can be expressed as $y_{3 t}^{+}=\sum_{i=1}^{t} \varepsilon_{3 i}^{+}, y_{3 t}^{-}=\sum_{i=1}^{t} \varepsilon_{3 i}^{-}$ and $y_{4 t}^{+}=\sum_{i=1}^{t} \varepsilon_{4 i}^{+}, y_{4 t}^{-}=\sum_{i=1}^{t} \varepsilon_{4 i}^{-}$. In the next step, causality among variables is determined by using the vector autoregressive model. For instance, positive cumulative shocks between variables can be tested by the following specification:

$y_{t}^{+}=\theta+\delta_{1} y_{t-1}^{+}+\ldots+\delta_{p} y_{t-1}^{+}+v_{t}^{+}$

where $y_{t}^{+}$denotes $2 \times 1$ vector of the variables, $\theta$ is $2 \times 1$ vector of the constant terms, and $v_{t}^{+}$is $2 \times 1$ vector of the residuals. $\delta_{r}$ represents $2 \times 2$ matrix of coefficients for the lag order $r(r=1, \ldots, p)$. The $\mathrm{VAR}_{p}$ model can be expressed as follows:

$Y=D Z+\lambda$

where $Y=\left(y_{1}^{+}, \ldots, y_{T}^{+}\right)(n x T)$ matrix, $D=\left(\theta, A_{1}, \ldots A_{p}\right)(n x(1+n p))$ matrix, $\lambda=\left(u_{1}^{+}, \ldots u_{T}^{+}\right)$, and $Z_{t}$ can be defined as follows:

$Z_{t}=\left[\begin{array}{c}1 \\ y_{t}^{+} \\ y_{t-1}^{+} \\ \cdot \\ \cdot \\ y_{t-p+1}^{+}\end{array}\right]((1+n p) x 1)$ matrix, for $t=1, \ldots T$,

where $Z=\left(Z_{0}, \ldots Z_{T-1}\right)((1+n p) x T)$ matrix. Finally, the null hypothesis of non-Granger causality $\left(H_{0}: C \varphi=0\right)$ can be tested by the following specification:

Wald $=(C \varphi)^{\prime}\left[C\left(\left(Z^{\prime} Z\right)^{-1} \otimes S_{U}\right) C^{\prime}\right]^{-1}(C \varphi)$

where $\varphi=\operatorname{vec}(D)$ and vec represent the column-stacking operator; $\otimes$ represents Kronecker product, $C$ is $\operatorname{pxn}(1+n p)$ indicator matrix, and $S_{U}$ represents covariance matrix of the unrestricted estimated VAR model. Besides, Hatemi-j (2012) conducted a bootstrap simulation with leverage adjustments to obtain more precise critical values.

We began our analysis by conducting Toda-Yamamoto and bootstrap-augmented Toda-Yamamoto causality test. According to the findings (Table 1), the null hypothesis of non-Granger
Table 1 Causality tests results

Toda-Yamamoto and bootstrap-augmented Toda-Yamamoto tests

Hypothesis Test stat Asymptotic Bootstrap prob $\boldsymbol{k} \rho$

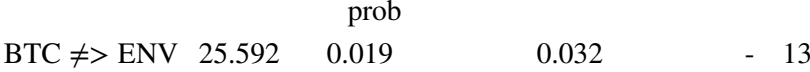

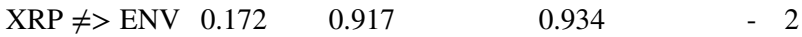

ETH $\neq>$ ENV $18.480 \quad 0.010 \quad 0.022 \quad-7$

Fourier Toda-Yamamoto test with single frequency

$\begin{array}{llllll}\text { Hypothesis } & \text { Test stat } & \begin{array}{c}\text { Asymptotic } \\ \text { prob }\end{array} & \text { Bootstrap prob } & \boldsymbol{k} & \rho \\ \text { BTC } \neq>\text { ENV } & 25.118 & 0.022 & 0.043 & 3 & 13 \\ \text { XRP } \neq>\text { ENV } & 2.973 & 0.085 & 0.094 & 1 & 1 \\ \text { ETH } \neq>\text { ENV } & 10.142 & 0.181 & 0.209 & 1 & 7\end{array}$

Fourier Toda-Yamamoto test with cumulative frequency

\begin{tabular}{llllll} 
Hypothesis & Test stat & $\begin{array}{c}\text { Asymptotic } \\
\text { prob }\end{array}$ & Bootstrap prob & $\boldsymbol{k}$ & $\rho$ \\
BTC $\neq>$ ENV & 24.714 & 0.025 & 0.045 & 3 & 13 \\
XRP $\neq>$ ENV & 9.829 & 0.020 & 0.060 & 3 & 3 \\
ETH $\neq>$ ENV & 5.195 & 0.636 & 0.657 & 3 & 7 \\
\hline
\end{tabular}

$\rho$, optimal lag, $k$, number of Fourier frequency. Optimal lag lengths were determined by using the Schwarz information criterion. Bootstrap critical values were obtained by utilizing 1000 bootstrap replications

causality running from XRP to environmental degradation is accepted, whereas null hypotheses of non-Granger causality running from BTC and ETH to environmental degradation are rejected. This implies causal effects running from BTC and ETH to environmental degradation, whereas no causal nexus is observed from XRP to environmental degradation.

The standard Toda-Yamamoto and bootstrap-augmented Toda-Yamamoto causality tests do not consider possible structural changes in the nature of the data. Enders and Jones (2016) reported that ignoring such structural shifts in series could lead to false rejection of the null of noncausality in the case of non-existence of causal linkages among variables. It can be observed in Fig. 1 that cryptocurrencies frequently exhibit volatile behaviors and sensitive to both economic and political events. Therefore, it is crucial to check the robustness of the estimations by employing a causality approach that controls structural changes in data. To this end, we employed Fourier TodaYamamoto proposed by Nazlioglu et al. (2016). The Fourier Toda-Yamamoto causality test with single and cumulative frequency results (Table 1) suggest the null hypothesis of non-Granger causality running from ETH to environmental degradation is accepted, while the null hypotheses of non-Granger causality running from BTC and XRP to environmental degradation are rejected. Thus, the assessment finds causal effects running from BTC and XRP to environmental degradation, whereas no causal nexus is observed from ETH to environmental degradation. 
The price fluctuations in financial markets can cause investors to act in several ways-in the case of price decrease, investors either stop-loss or take long position whereas in the case of price increase, investors either take profits or invest more. Hatemi-j (2012) noted investors could respond differently to positive shocks compared to negative shocks of absolute magnitudes in the financial market. In this manner, investment behavior is more sensitive to negative shocks than positive ones. Therefore, asymmetric causal effects could occur in financial markets. Besides, the existence of asymmetric information on financial markets can cause the occurrence of asymmetric causal effects (Stiglitz 1974; Akerlof 1978; Hatemi-j 2012). It can be said that cryptocurrencies remain one of the markets with asymmetric information. Business Insider (2021a) reported nearly 1000 individuals known as "whales," who hold almost $40 \%$ of the cryptocurrency market-easily manipulating cryptocurrency valuation, hence, causing serious price fluctuations. In such a case, the investment decisions of ordinary investors can easily be manipulated-by taking long position after selling huge amount of cryptocurrencies and vice versa; hence, small investors easily make a loss due to asymmetric information of behaviors of whales in cryptocurrency market. Thus, asymmetric causal effects may easily occur in cryptocurrency market. To consider asymmetries in cryptocurrency market, we utilized an asymmetric causality test to investigate the effects of positive and negative demand shocks of cryptocurrencies on the environment.

The asymmetric causality test results (Table 2) validate the null hypothesis of non-Granger causality from positive shocks of BTC to positive shocks of environmental degradation (i.e., $p$-value $<0.10$ ) is rejected, whereas the null hypotheses of non-Granger causality are accepted for from positive shocks of BTC to negative shocks of environmental degradation, and from negative shocks of BTC to negative and positive shocks of environmental degradation, respectively. Therefore, positive shock in BTC demand causes positive shocks to environmental pollution. Moreover, the null hypothesis of non-Granger causality from negative shocks of XRP and ETH to positive shocks of environmental degradation is rejected at $1 \%$ and 5\% significance levels, respectively, whereas the null hypothesis of non-Granger causality is accepted for other three hypotheses regarding XRP-ETH and environmental degradation. Thus, negative shocks in XRP and ETH demand cause positive shocks to environmental pollution.

\section{Discussion}

Cryptocurrencies are regarded as decentralized, secure, low-cost payment tools for financial transactions. Therefore, it is asserted that the value of any cryptocurrency
Table 2 Asymmetric causality test results

\begin{tabular}{lcrrr}
\hline & & \multicolumn{3}{c}{ Bootstrap Critical Values } \\
\cline { 3 - 5 } Hypothesis & Test Stat & \multicolumn{1}{c}{$1 \%$} & \multicolumn{1}{c}{$5 \%$} & \multicolumn{1}{c}{$\%$} \\
\hline $\mathrm{BTC}^{+} \neq>\mathrm{ENV}^{+}$ & $5.516^{*}$ & 9.697 & 6.030 & 4.497 \\
$\mathrm{BTC}^{+} \neq>\mathrm{ENV}^{-}$ & 1.217 & 9.483 & 6.295 & 4.626 \\
$\mathrm{BTC}^{-} \neq>\mathrm{ENV}^{-}$ & 0.529 & 9.041 & 3.904 & 2.743 \\
$\mathrm{BTC}^{-} \neq>\mathrm{ENV}^{+}$ & 0.288 & 10.860 & 6.672 & 5.153 \\
$\mathrm{XRP}^{+} \neq>\mathrm{ENV}^{+}$ & 1.064 & 11.296 & 4.446 & 2.918 \\
$\mathrm{XRP}^{+} \neq>\mathrm{ENV}^{-}$ & 5.066 & 25.771 & 15.468 & 12.355 \\
$\mathrm{XRP}^{-} \neq>\mathrm{ENV}^{-}$ & 0.101 & 12.535 & 4.881 & 3.012 \\
$\mathrm{XRP}^{-} \neq>\mathrm{ENV}^{+}$ & $39.564 * * *$ & 27.519 & 16.849 & 13.753 \\
$\mathrm{ETH}^{+} \neq>\mathrm{ENV}^{+}$ & 15.020 & 154.623 & 64.736 & 38.166 \\
$\mathrm{ETH}^{+} \neq>\mathrm{ENV}^{-}$ & 11.528 & 36.993 & 18.456 & 14.056 \\
$\mathrm{ETH}^{-} \neq>\mathrm{ENV}^{-}$ & 0.004 & 13.575 & 5.281 & 2.845 \\
$\mathrm{ETH}^{-} \neq>\mathrm{ENV}^{+}$ & $37.398 * *$ & 43.308 & 22.681 & 16.390 \\
\hline
\end{tabular}

Optimal lag lengths were determined by using Akaike information criterion $* * *$, and $* * *$ show statistical significance at 10,5 , and $1 \%$ level, respectively. Critical values were obtained by utilizing 1000 bootstrap replications

could be determined by the market forces without exogenous intervention such as central banking policy. The idea of decentralized currency has increasingly been adopted by individuals, hence, increasing cryptocurrency demand and mining activities across the globe. Besides, the adoption of cryptocurrencies is reported to have increased during the global pandemic, viz. COVID-19-when social distancing measures were enforced (Sarkodie et al. 2021). However, the environmental cost of cryptocurrency markets is often ignored in the existing literature.

In this study, we aimed to unveil how cryptocurrency demand affects environmental sustainability via empirical assessment. The empirical results provide broad insights into the cryptocurrency market and environmental quality interaction. The standard causality test results suggest the existence of causal effects running from cryptocurrency demand to environmental degradation, but unable to separate the causal impact of positive and negative shocks of Bitcoin demand on environmental degradation (Hatemi-J 2012). To address this issue, we utilized an asymmetric causality approach. The results suggest positive shocks on BTC and negative shocks on XRP and ETH demand predict environmental degradation. First, this might be linked to increasing number of cryptocurrency transactions and mining activities during the period of volatility - either for having speculative profit or avoiding loss of investment-a typical intuition of cryptocurrency investors. Second, due to future expectations of price and revenue increase as same as BTC, negative price shocks on alternative cryptocurrencies such as XRP and ETH may induce investors to demand and increase mining activities of these alt coins (Katsiampa 2017). Third, due 
to unregulated crypto market, transactions could be done with nearly low or no cost without taxes (Stone 2021). In such a case, the number of cryptocurrency transactions may easily increase, hence, inevitably inducing more energy consumption from fossil fuels - with long-term implications on environmental pollution. For instance, due to the nature of blockchain technology, a huge number of third parties are involved to make a BTC transaction even if it includes lower quantities of cryptocurrency. In this process, the existence of related cryptocurrency is verified by blockchain technology through controlling formerly recorded blocks-verifying the accuracy of a new transaction and recording unalterable records on the accounts of parties existing in the network (Martin and Nauman 2021).

Unlike XRP with more energy-efficient solution, both BTC and ETH are based on proof-of-work (PoW) consensus mechanism which is energy-dependent. However, the BTC network is energy-intensive compared to ETH. It is estimated that the annualized total BTC carbon footprint of 93.80 $\mathrm{MtCO}_{2}$ can be compared to Nigeria's carbon footprint (Digiconomist 2021a, b). The annualized total electrical energy of BTC, viz. 197.47 TWh is comparable to Thailand's power consumption whereas resulting electronic waste $(27.64 \mathrm{kt})$ from BTC mining can be compared to the Netherland's IT wastes (Digiconomist 2021a). A recent study showed that BTC's annual e-waste generated could exceed 30.7 kt (De Vries and Stoll 2021). Contrary, Ethereum's estimated annualized total carbon footprint of 43.56 $\mathrm{MtCO}_{2}$ can be compared to Hong Kong's carbon footprint, whereas its annualized total electrical energy of 91.7 TWh is comparable to the Philippines' power consumption (Digiconomist 2021b). In comparison, a single BTC transaction entails $1955.06 \mathrm{kWh}$ electrical energy, $928.65 \mathrm{kgCO}_{2}$ carbon footprint, and $273.70 \mathrm{~g}$ of e-waste equivalent to average US household power use for 67 days, 154,775 YouTube watch hours, and weight of $0.56 \mathrm{iPad}$, respectively--whereas a single ETH transaction comprises $194.16 \mathrm{kWh}$ electrical energy and $92.23 \mathrm{kgCO}_{2}$ carbon footprint similar to average US household power use for 6.56 days and 204,414 VISA transactions, respectively (Digiconomist, 2021a,b).

If it is considered that high-performance computers such as gaming computers are widely used in cryptocurrency markets, the environmental results of cryptocurrency demand could be more dramatic. Mills and Mills (2016) reported the average electricity consumed by gaming computers as $\sim 1,394$ kilowatt-hours (kWh)/year. This implies gaming computer consumes an average of $3.82 \mathrm{kWh}$ of electricity per day. Business Insider (2021b) reported that the number of cryptocurrency users surpassed 100 million by February, 2021. Thus, 1-h cryptocurrency trade of 100 million investors is almost equal to $15,913,242.01 \mathrm{kWh} /$ day and 5,808,333,333.33 kWh/year electricity consumption. This can further be estimated in terms of emissions by using the estimation method of Sibelga (2021)where the amount of electricity consumption is nearly equal to $1,694,097,222.22 \mathrm{~kg} /$ year carbon emissions, viz. 1,694,097.22 metric tons/year of $\mathrm{CO}_{2}$ emissions. This infers each cryptocurrency investor emits $\sim 0.0169$ metric tons/per capita year of $\mathrm{CO}_{2}$ emissions-assuming one-hour daily cryptocurrency trade. The emission value is almost equal to $0.37 \%$ of global average $\mathrm{CO}_{2}$ emissions per capita (metric tons). In another scenario, if cryptocurrencies are traded on the digital platforms by using mobile phones, tablet PCs, and laptops, the amount of $\mathrm{CO}_{2}$ emission could increase (Martin and Nauman 2021).

A similar approach can be adopted for $\mathrm{CO}_{2}$ emissions emitted from cryptocurrency mining activities. According to statistics from Slushpool (2021), the world's first BTC mining pool, there are approximately 147,500 BTC miners actively working. Assuming mining activities are executed on full-time basis, 1 day of mining BTC by $\sim 147,500$ miners is almost equal to $563,328.77 \mathrm{kWh} /$ day and 205,615,000.00 kWh/year electricity consumption. Similarly, the estimated amount of electricity consumption is nearly equal to $59,971,041.67 \mathrm{~kg} /$ year of $\mathrm{CO}_{2}$ emissions, or 59,971.04 metric tons/year $\mathrm{CO}_{2}$ emissions from only one cryptocurrency-mining activity. This means each BTC-miner emits almost 0.406 metric tons/ year $\mathrm{CO}_{2}$ emissions per capita, which is almost equal to $8.92 \%$ of global average $\mathrm{CO}_{2}$ emissions per capita (metric tons). CoinMarketCap (2021) reports more than 14,812 (est. November 25, 2021) different cryptocurrencies in circulation, thus, $\mathrm{CO}_{2}$ emissions emitted by cryptocurrency mining activities and transactions could dramatically increase.

Though most countries are suffering from lack of legislation on cryptocurrency markets, however, the statistics provided implies immediate action required to regulate cryptocurrency markets at the global level to internalize the environmental externalities associated with cryptocurrency activities. Considering the influx of cryptocurrency mining revenues (Fig. 3), overshooting of cryptocurrency prices, unusual profits, and expectations related to increasing cryptocurrency values in the future; due to high level of energy use, the environmental cost of crypto market has the potential to exhibit a sharp increase (University of Cambridge 2021). Additionally, the number of cryptocurrencies and its corresponding users are increasing dayby-day, thus, policymakers could immediately focus on diminishing the environmental burden of cryptocurrency mining activities and transactions at national and international levels. Failure to regulate the budding potential of cryptos could thwart the environmental aims determined by international initiatives such as Kyoto Treaty, Paris Summit, Sustainable Development Goals, hence, affecting climate change. 
Fig. 3 Revenue raised from cryptocurrency mining worldwide. Source: Statista (2021)

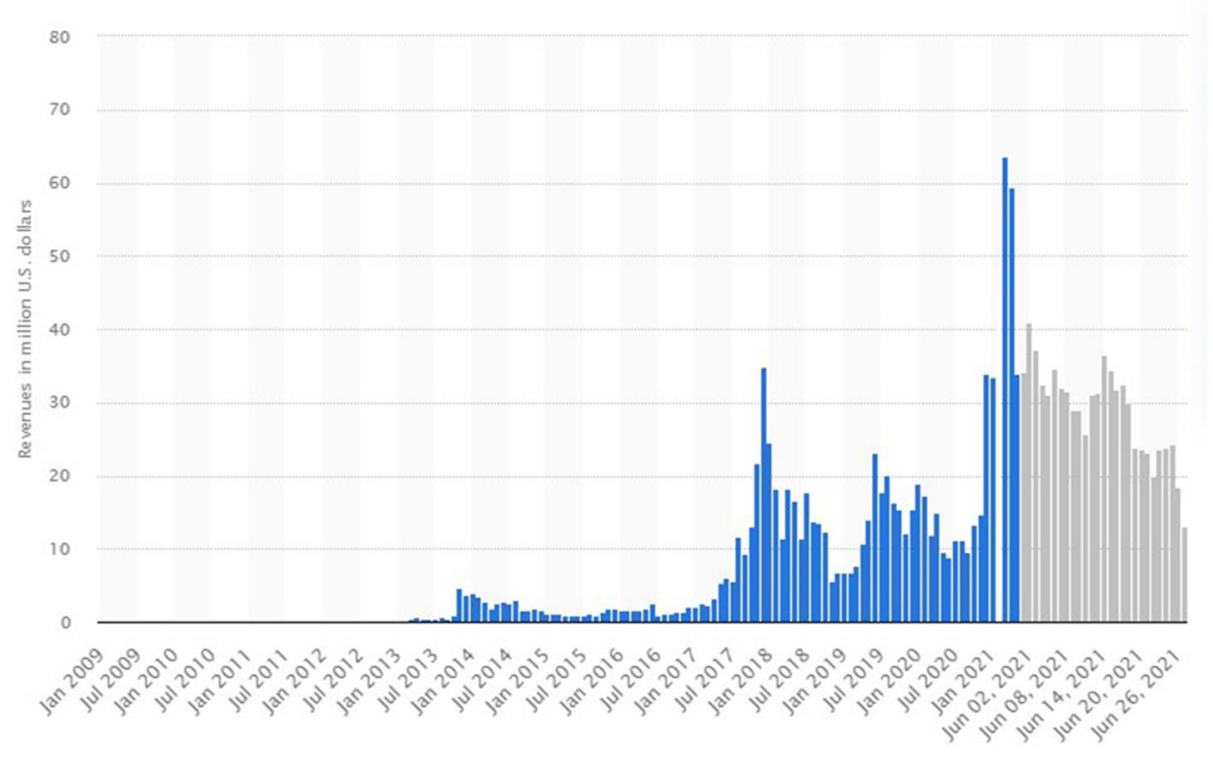

\section{Conclusion and policy implications}

In this study, we investigated whether the causal effects run from cryptocurrency demands to environmental degradation by employing a set of symmetric and asymmetric causality tests. According to the symmetric causality test results, cryptocurrency demand has causal effects on environmental degradation. The asymmetric causality test results indicate that there are asymmetric causal effects from cryptocurrency demand to environmental degradation.

The results reveal the exigency of policy implementations on cryptocurrency markets. First, politicians could immediately focus on detecting whether characteristics of symmetric causal effects are negative or positive. Second, policymakers could pay attention to controlling the causal effects of cryptocurrency demand on environmental quality. In this manner, establishing financial and environmental legislation to regulate the cryptocurrency market might be an effective way. Third, to control the effects of pollution-enhancer asymmetric shocks of cryptocurrency demand, policymakers could consider imposing an environmental tax on cryptocurrency transactions and revenues. This may help reduce the environmental burden of cryptocurrency trade by preventing speculative profit expectations from crypto markets. Fourth, policymakers could pay attention to formalizing cryptocurrency transactions by establishing legal procedures. Within this regard, the license implementation on cryptocurrency trade could be an option, and it will allow controlling environmental effects of cryptocurrency trade, whereas environmental taxes could help the internalization of the negative externalities caused by mining activities and transactions in the scope of "polluter pays" principle. Finally, based on the aims and objectives of Industry 4.0, a paradigm shift from PoW consensus mechanism to green alternatives, typically proof-of-stake could improve energy consumption efficiency, thereby reducing carbon footprint of PoW-based cryptocurrencies.

Though this paper was based on novel and robust estimation techniques, yet, has several limitations. Due to the lack of data on cryptocurrencies, we could not include widely known cryptocurrencies into the analysis. The cryptocurrency market is experiencing unprecedented growth and number of cryptocurrencies increasing day-by-day. Therefore, future studies may consider including other cryptocurrencies in the analysis. Moreover, due to existence of relatively small number of data observation, we did not conduct an analysis to make inferences for the long-run such as cointegration and cointegration estimation. Therefore, future studies could focus on the magnitude of long-run effect of cryptocurrency demand on the environment. Last, it is widely known that the size and power properties of empirical methods increase with time dimension, hence, future studies may consider analyzing with a longer time span.

Author contributions SE: conceptualization, formal analysis, writingoriginal draft, writing - review and editing, MYA: investigation, writing—original draft; writing — review and editing, SAS: visualization, supervision, writing —original draft; writing—review and editing.

Data availability The datasets used and/or analyzed during the current study are available from the corresponding author on reasonable request. 


\section{Declarations}

Competing interests The authors declare no competing interests.

\section{References}

Akalin G, Erdogan S, Sarkodie SA (2021) Do dependence on fossil fuels and corruption spur ecological footprint? Environ Impact Assess Rev 90:106641

Akerlof GA (1978) 15 - The market for "lemons": Quality uncertainty and the market mechanism. In: Diamond P, Rothschild M (eds) Uncertainty in Economics. Academic Press, pp 235-251.https:// doi.org/10.1016/B978-0-12-214850-7.50022-X

Badea L, Mungiu-Pupăzan MC (2021) The economic and environmental impact of bitcoin. IEEE Access 9:48091-48104

Baur DG, Oll J (2021) Bitcoin investments and climate change: a financial and carbon intensity perspective. Finance Research Letters, 102575. https://doi.org/10.1016/j.frl.2021.102575

Business Insider (2021a). Bitcoin whales: what are they - and how are they affecting the cryptocurrency's price? Retrieved from https:// buff.ly/3ro8mpJ. Accessed 04.03.2021.

Business Insider (2021b). The estimated number of global crypto users has passed 100 million - and boomers are now getting drawn to Bitcoin too, reports find. Retrieved from https://buff.ly/3nPZw1s. Accessed 07.03.2021.

CoinMarketCap (2021). Today's cryptocurrency prices by market cap. Retrieved from https://coinmarketcap.com/. Accessed 25.11.2021.

De Vries A, Stoll C (2021) Bitcoin's growing e-waste problem. Resour Conserv Recycl 175:105901

Danish, Ulucak R (2020) The pathway toward pollution mitigation: does institutional quality make a difference? Bus Strateg Environ 29(8):3571-3583

Digiconomist (2021a). Bitcoin energy consumption index. Retrieved from https://buff.ly/2hpcEYa. Accessed 25.11.2021.

Digiconomist (2021b). Ethereum energy consumption index. Retrieved from https://buff.ly/2SM5vD4. Accessed 25.11.2021.

Elon, M. (2021). Tesla \& bitcoin. Retrieved from https://buff.ly/33BGN Mr. Accessed 07.03.2021.

Enders W, Jones P (2016) Grain prices, oil prices, and multiple smooth breaks in a VAR. Stud Nonlinear Dyn Econom 20(4):399-419

Erdogan S, Akalin G, Oypan O (2020) Are shocks to disaggregated energy consumption transitory or permanent in Turkey? New evidence from fourier panel KPSS test. Energ 197:117174

Erdogan S (2020) Analyzing the environmental Kuznets curve hypothesis: the role of disaggregated transport infrastructure investments. Sustain Cities Soc 61:102338

Garcia D, Tessone CJ, Mavrodiev P, Perony N (2014) The digital traces of bubbles: feedback cycles between socio-economic signals in the Bitcoin economy. J R Soc Interface 11(99):20140623

Global Monitoring Laboratory (2021). GML data finder. https://buff. ly/3CQQMwo. Accessed 01.02.2021.

Goodkind AL, Jones BA, Berrens RP (2020) Cryptodamages: monetary value estimates of the air pollution and human health impacts of cryptocurrency mining. Energ Res Soc Sci 59:101281

Grossman GM, Krueger AB (1991) Environmental impacts of a North American free trade agreement. National Bureau of Economic Research. Working paper no. w3914

Hacker RS, Hatemi-J A (2006) Tests for causality between integrated variables using asymptotic and bootstrap distributions: theory and application. Appl Econ 38(13):1489-1500

Hatemi-j A (2012) Asymmetric causality tests with an application. Empirical Economics 43(1):447-456
Hayes AS (2017) Cryptocurrency value formation: an empirical study leading to a cost of production model for valuing bitcoin. Telematics Inform 34(7):1308-1321

Ilkay SC, Yilanci V, Ulucak R, Jones K (2021) Technology spillovers and sustainable environment: evidence from time-series analyses with Fourier extension. J Environ Manag 294:113033

Investing (2021). Cryptocurrency. https://www.investing.com/crypto/. Accessed 01.02.2021 2021.

Jiang S, Li Y, Lu Q, Hong Y, Guan D, Xiong Y, Wang S (2021) Policy assessments for the carbon emission flows and sustainability of Bitcoin blockchain operation in China. Nat Commun 12(1):1-10

Kahn B (2018) Mining Bitcoin can be more of an energy drain than actual mining climate change. Retrieved from https://buff.ly/2ztUc $\mathrm{Xb}$. Accessed 07.03.2021.

Katsiampa P (2017) Volatility estimation for Bitcoin: a comparison of GARCH models. Econ Lett 158:3-6

Kosba A, Miller A, Shi E, Wen Z, Papamanthou C (2016) Hawk: the blockchain model of cryptography and privacy-preserving smart contracts. Paper presented at the 2016 IEEE symposium on security and privacy (SP)

Krause MJ, Tolaymat T (2018) Quantification of energy and carbon costs for mining cryptocurrencies. Nature Sustainability 1(11):711-718

Martin K, Nauman B (2021) Bitcoin's growing energy problem: 'It's a dirty currency'. Retrieved from https://buff.ly/3hELddz. Accessed 09.06.2021.

McGeeham P (2018) Bitcoin miners flock to New York's remote corners, but get chilly reception. Retrieved from https://buff.ly/ 2DdkwKy. Accessed 07.03.2021

Mills N, Mills E (2016) Taming the energy use of gaming computers. Energ Effi 9(2):321-338

Mohsin K (2021) Cryptocurrency \& Its Impact on Environment. Available at SSRN 3846774

Mohsin M, Naseem S, Zia-ur-Rehman M, Baig SA, Salamat S (2020) The crypto-trade volume, GDP, energy use, and environmental degradation sustainability: an analysis of the top 20 crypto-trader countries. Int J Financ Econ. https://doi.org/10.1002/ijfe.2442

Mora C, Rollins RL, Taladay K, Kantar MB, Chock MK, Shimada M, Franklin EC (2018) Bitcoin emissions alone could push global warming above $2 \mathrm{C}$. Nat Clim Chang 8(11):931-933

Nakamoto S (2008) Bitcoin: A peer-to-peer electronic cash system. Decentralized Business Review, 21260. Retrieved from https:// buff.ly/2uy0oyr. Accessed 09.06.2021

Nazlioglu S, Gormus NA, Soytas U (2016) Oil prices and real estate investment trusts (REITs): gradual-shift causality and volatility transmission analysis. Energy Econ 60:168-175

Patil I (2021) Visualizations with statistical details: The 'ggstatsplot' approach. J Open Source Softw, 6(61)

Peck ME (2017) Why the biggest Bitcoin mines are in China. Retrieved from https://buff.ly/3cNmz6X. Accessed 09.06.2021

Rowlatt J (2020) How Bitcoin's vast energy use could burst its bubble. Retrieved from https://buff.ly/3uBArsu. Accessed 09.06.2021

Sarkodie SA, Ahmed MY, Owusu PA (2021) COVID-19 pandemic improves market signals of cryptocurrencies-evidence from Bitcoin, Bitcoin Cash, Ethereum, and Litecoin. Financ Res Lett. 102049. https://doi.org/10.1016/j.frl.2021.102049

Schinckus C, Nguyen CP, Ling FCH (2020) Crypto-currencies trading and energy consumption. Int J Energ Econ Policy 10(3):355

Sibelga (2021) How much power does a computer use? And how much $\mathrm{CO}_{2}$ does that represent? Retrieved from https://buff.ly/2Zm4slo. Accessed 09.06.2021

Sinha A, Shahbaz M, Balsalobre D (2019) Chapter 7 - Data selection and environmental kuznets curve models - environmental kuznets curve models, data choice, data sources, missing data, balanced and unbalanced panels. In: Özcan B, Öztürk I (eds) Environmental 
Kuznets Curve (EKC). Academic Press, pp 65-83.https://doi.org/ 10.1016/B978-0-12-816797-7.00007-2

Slushpool (2021) Pool statistics. Retrieved from https://buff.ly/3p2w2 Nd. Accessed 07.03.2021

Statista (2021) Revenue raised from cryptocurrency mining worldwide from January 2009 to February 15, 2021. Retrieved from https:// buff.ly/3nQ1b7c. Accessed 08.03.2021

Stiglitz JE (1974) Incentives and risk sharing in sharecropping. Rev Econ Stud 41(2):219-255

Stoll C, Klaaßen L, Gallersdörfer U (2019) The Carbon Footprint of Bitcoin. Joule 3(7):1647-1661

Stone S (2021) 2021 Crypto-exchange fee comparison. Retrieved from https://buff.ly/318LQwN. Accessed 09.06.2021
Toda HY, Yamamoto T (1995) Statistical inference in vector autoregressions with possibly integrated processes. Journal of Econometrics 66(1-2):225-250

University of Cambridge (2021) Cambridge Bitcoin electricity consumption index. Retrieved from https://cbeci.org. Accessed 08.06.2021.

Welch BL (1951) On the comparison of several mean values: an alternative approach. Biometrika 38(3/4):330-336

Publisher's Note Springer Nature remains neutral with regard to jurisdictional claims in published maps and institutional affiliations. 